\title{
LA INTERVENCIÓN HUMANITARIA COMO MECANISMO DE PROTECCIÓN DE LOS DERECHOS FUNDAMENTALES EN LA SOCIEDAD INTERNACIONAL
}

\author{
Antonio Manrique de Luna Barrios \\ Doctor en Derecho Internacional, Máster en Estudios Internacionales, Máster en Comercio Exterior, Licenciado \\ en Derecho Español y Peruano, B.A. en Ciencias Políticas. Profesor de Derecho en la Universität Osnabrück \\ (Alemania). \\ E: mail: amanriqu@uni-osnabrueck.de
}

\begin{abstract}
Resumen
En la perspectiva actual, la humanidad ha decidido desde fines del siglo XX, el proclamar su sentimiento de pertenencia a una humanidad única, sin fronteras y en virtud de la cual se permite en nombre de "todos" interferir en la soberanía de cada Estado, cuando están de por medio la paz, la seguridad internacional y la vigencia de los derechos fundamentales.

Habida cuenta de ello, la prevención y la resolución de los conflictos armados han pasado a constituir una de las tareas más apremiantes que tiene ante sí la Sociedad Internacional y que han situado a la Intervención Humanitaria, en el centro de un intenso debate en el que se ha puesto en tela de juicio su naturaleza, sus métodos y su propia razón de ser frente a las guerras contemporáneas.
\end{abstract}

Palabras Clave: Intervención humanitaria, derechos fundamentales, paz, seguridad internacional, Sociedad Internacional, soberanía.

\begin{abstract}
At the moment, beginning from the end of the 20th century humanity has decided to proclaim a certain sense of belonging to one humanity without borders in which it is permitted to interfere with the sovereignty of each State in the name of "everyone", when the subjects are means of peace, international security and the validity of the fundamental rights.

Taking this into consideration the resolution of armed conflicts has become one of the most urgent tasks that the International Society is faced with and has placed the humanitarian intervention in the centre of an intense debate in which is questioned its nature, its methods and its own reasons in respect to the contemporary wars.
\end{abstract}

Key Words: Humanitarian intervention, fundamental rights, peace, international security, International Society, sovereignty

\section{Résumé}

Dans la perspective d'aujourd'hui, l'humanité a décidé depuis le fin du XXe siècle, en proclamant leur sentiment d'appartenance à une humanité unique, sans frontières et le nom sous lequel est autorisé dans «tous» interférer dans la souveraineté de chaque État, quand ils sont dans la paix, la sécurité internationale et le respect des droits fondamentaux.

Compte tenu de cela, la prévention et la résolution des conflits armés sont venus à constituer l'une des tâches les plus urgentes devant elle et la Société internationale ont placé l'intervention humanitaire dans le centre d'un intense débat dans lequel a été mis en cause son caractère, ses méthodes et sa propre raison d'être contre les guerres contemporaines.

Mots-clés: l'intervention humanitaire, les droits fondamentaux, la paix, la sécurité internationale, la Société internationale, la souveraineté. 

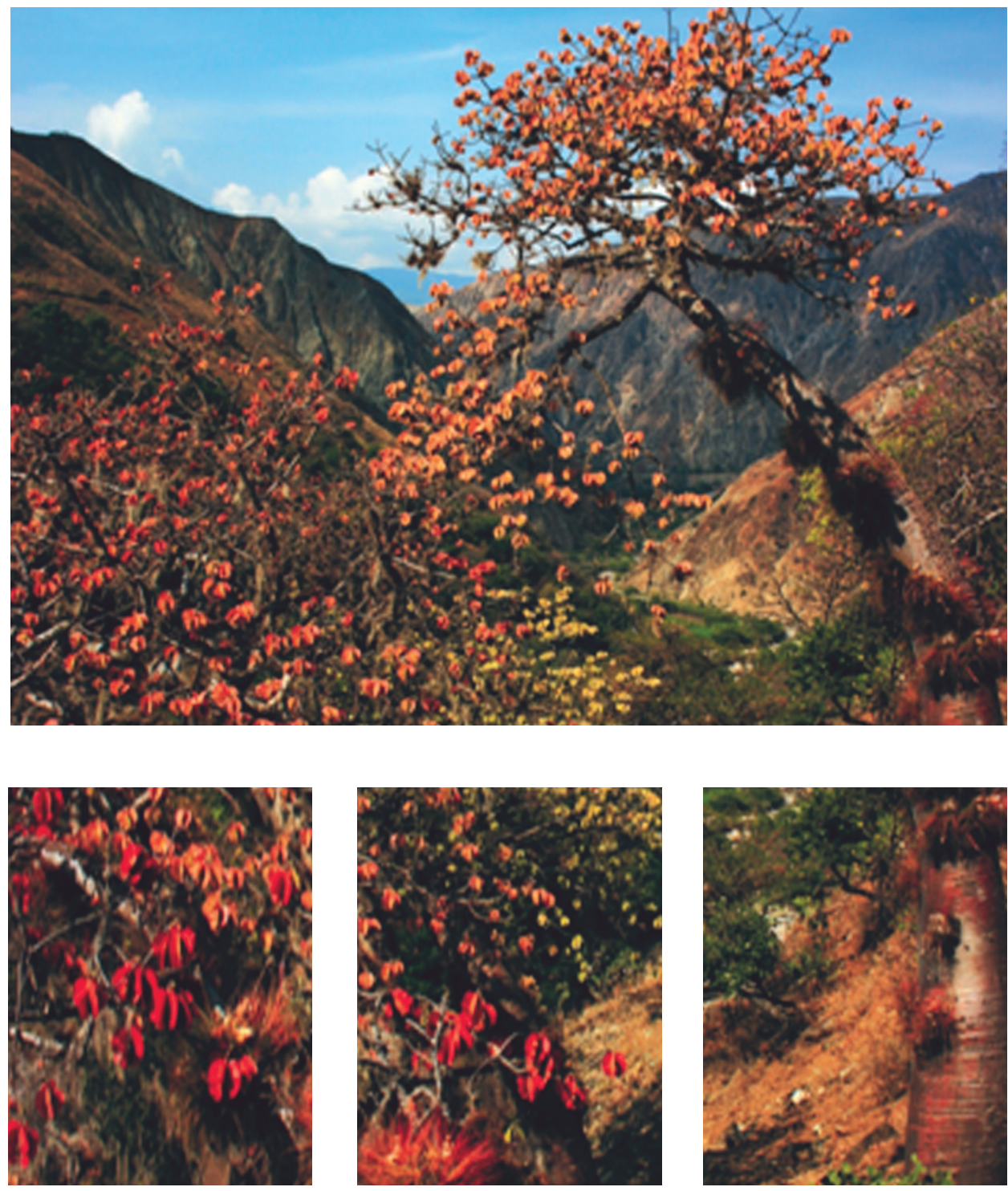

Cañón del Chicamocha - Ceibas Barrigonas

Jorge William Sánchez Latorre 


\section{LA INTERVENCIÓN HUMANITARIA COMO MECANISMO DE PROTECCIÓN DE LOS DERECHOS FUNDAMENTALES EN LA SOCIEDAD INTERNACIONAL*}

Antonio Manrique de Luna Barrios

\section{INTRODUCCIÓN}

La intervención humanitaria, puede ser entendida como una acción de la Sociedad Internacional dentro de un determinado país, cuando se producen ahora graves vulneraciones de los derechos fundamentales y/o existe un gran riesgo de que determinados grupos de la población puedan sucumbir o desaparecer ante esas prácticas sistemáticas que vulneran sus derechos fundamentales.

Indudablemente, dicha actuación de los Estados miembros de la Sociedad Internacional debe darse de acuerdo a situaciones objetivas que fundamenten la realización de dicha actividad. En el escenario internacional las relaciones entre los diferentes Estados deben estar enmarcadas en un respeto mutuo y en la no intervención en los asuntos internos que se produzcan dentro de cada Estado. Sin embargo, al producirse vulneraciones de los derechos fundamentales dentro de un Estado, la Sociedad Internacional puede intervenir para frenar dichos actos que son contrarios al ordenamiento jurídico internacional. En tal sentido, el rol de la Organización de las Naciones Unidas, de las organizaciones regionales y de los Estados que lideran fuerzas multinacionales es de gran importancia y resulta un soporte fundamental para proteger a la población civil que se encuentra en dichos lugares.

El presupuesto del que partimos en la presente investigación es que las intervenciones humanitarias, han permitido que se puedan llevar a cabo una serie de actividades con la finalidad de proteger los derechos fundamentales. Sin embargo,

* En la presente investigación se revisa acerca de la intervención humanitaria, como mecanismo de protección de los derechos fundamentales en el marco de la sociead internacional, abordado desde un método interdisciplinar que permite analizar la implementación práctica de intervenciones desarrolladas en algunos paises, desde el marco investigativo señalado por el autor. 
dichas actividades no siempre han estado exentas de críticas en la medida que se ha permitido diversas actuaciones que constantemente se encuentran en la frontera entre la legalidad y la ilegalidad.

En ese sentido, el objeto de esta investigación es precisar en qué consiste una intervención humanitaria y qué elementos deben estar presentes para configurar una actuación de ese tipo, ya que muchas veces se pretende amparar un simple acto de intervención bajo el amparo de que se busca proteger los derechos fundamentales de la población de un determinado país. En virtud de ello, en el presente estudio, se analiza la conceptualización de la intervención humanitaria, con la finalidad de establecer cuál es la idea central con la que se debe identificar a dicha actividad que se lleva a cabo en el escenario internacional. Así mismo, en el segundo apartado de este análisis, se estudiará la legalidad de las intervenciones humanitarias, con la finalidad de establecer qué requisitos deben darse para poder indicar que estamos frente a una real y objetiva intervención que busca proteger los derechos fundamentales. Por su parte, en el tercer apartado de la presente investigación, se estudiará la finalidad protectora de los derechos fundamentales que debe tener toda intervención humanitaria, con el objeto de evitar que actos como el racismo, la deportación en masa de colectivos humanos, entre otros aspectos, queden impunes ante la complicidad de las instituciones internas de un determinado Estado.

Así mismo, en el apartado cuarto de la presente investigación, se abordará la controversia que surge al enfrentar el principio de no intervención en los asuntos internos de otros países con las actuaciones que en el seno de la Sociedad Internacional que se han denominado como intervenciones humanitarias. Por su parte, en el apartado quinto, se estudiará la responsabilidad colectiva de los Estados en la implementación de las intervenciones humanitarias, con la finalidad de establecer que las vulneraciones graves de los derechos fundamentales y del derecho humanitario durante un conflicto armado, puede representar una amenaza grave para la paz y seguridad internacional que constituye un reclamo esencial para que los Estados miembros de la Sociedad Internacional puedan llevar a cabo una intervención de carácter humanitario. Posteriormente, en el apartado sexto, se desarrollará la responsabilidad de proteger como fundamento de una intervención humanitaria, dándose especial importancia a la responsabilidad de prevenir, a la responsabilidad de reaccionar y a la responsabilidad de reconstruir. Finalmente, en el apartado séptimo, se analizará de manera breve la implementación práctica de las intervenciones humanitarias, con la finalidad de analizar las experiencias en Irak, Haití, la ex- Yugoslavia, Ruanda, Kosovo y Libia.

A través de la presente investigación se pretende: 1) establecer un concepto claro respecto de lo que se debe entender por intervención humanitaria; 2) incentivar el debate en torno a las intervenciones humanitarias; 3) presentar un enfoque que permita frenar o evitar la manipulación de dicha actuación legalizada en el ámbito internacional cuando en realidad se desea simplemente intervenir en asuntos internos de otro país; 4) precisar qué actuaciones pueden ser consideradas como 
intervenciones humanitarias y cuáles no al amparo del ordenamiento jurídico internacional y especialmente de las normas de la Carta de las Naciones Unidas; 5) difundir las experiencias positivas y negativas en torno a la materia de estudio.

Se debe indicar que la presente investigación ha empleado un método multidisciplinar, en donde se combina la teoría y la práctica en torno a dicha materia. Por su parte, en cuanto a las fuentes empleadas, se ha consultado la doctrina internacional, la jurisprudencia internacional y diversas normas internacionales.

En el siguiente apartado se analizará el proceso de conceptualización de las intervenciones humanitarias que se ha llevado a cabo en el escenario internacional a lo largo del tiempo.

\section{LA CONCEPTUALIZACIÓN DE LA INTERVENCIÓN HUMANITARIA}

Tras las graves vulneraciones de los derechos fundamentales que se han producido en Libia, la Sociedad Internacional ha desarrollado una serie de acciones y medidas que se enmarcan dentro de lo que se ha considerado como la más grande intervención humanitaria del presente siglo. Tomando en cuenta ello, resulta necesario el establecer con claridad en qué consiste tal medida.

En primer lugar, se puede identificar a la intervención humanitaria con

"el derecho de un Estado a ejercer control internacional sobre los actos de otro en lo que atañe a su soberanía internacional cuando dichos actos contravienen las leyes de la humanidad."

En segundo lugar, se puede identificar a la intervención humanitaria como "la acción coercitiva, incluida la utilización de la fuerza armada, que emprenden determinados Estados en otro Estado sin el consentimiento del gobierno de éste, con o sin la autorización del Consejo de Seguridad de las Naciones Unidas, con el fin de prevenir o poner fin a violaciones graves y masivas de los derechos fundamentales o del derecho internacional humanitario." ${ }^{2}$

Al respecto, se debe señalar que si bien la intervención humanitaria no goza aún de una aceptación unánime, algunos tratadistas, la empiezan a ver como una Costumbre Internacional, que empieza a cristalizarse en el ámbito de la Sociedad Internacional. Sin embargo, desde nuestro punto de vista, rechazamos ese enfoque, por cuanto

1 Francis Kofi, The Evolution of the Doctrine and Practice of Humanitarian Intervention, Kluwer Law International. 1999. p. 3

2 Danish Institute of International Affairs, Humanitarian Intervention, Legal and Political Aspects, DUPI, 1999. p.11 
consideramos que aún no existe un consenso en la Sociedad Internacional respecto de dicha acto, que refleje un modelo claro de lo que la intervención humanitaria es. Tomado ello en consideración, se puede señalar que la intervención humanitaria tal como se ha llevado a cabo, se identifica más con una intervención armada de carácter programado. Situación plenamente condenable y que vendría a ser contradictoria con la Jurisprudencia que la Corte Internacional de Justicia ha desarrollado respecto del caso Nicaragua, donde se admitió que la asistencia humanitaria no tenía carácter de intervención condenable si se limitaba a los fines consagrados por la práctica del Comité Internacional de la Cruz Roja.

Sobre el particular, el Comité Internacional de la Cruz Roja, respecto de las intervenciones humanitarias, ha establecido que el Consejo de Seguridad de las Naciones Unidas no solamente debe autorizar el despliegue de una fuerza de intervención por razones humanitarias, sino que, además, debe velar por la protección de los derechos fundamentales de las personas que se encuentran en aquella zona donde se lleve a cabo la intervención que ha autorizado. No obstante ello, establece que la intervención humanitaria, implica una renuncia a prevenir los conflictos y promover los valores del Derecho Internacional Humanitario.

A continuación se analizarán los diversos requisitos que deben estar presentes en toda intervención para que podamos considerarla como de carácter humanitario $\mathrm{y}$, por ende, dentro de ciertos parámetros de legalidad dentro del ámbito internacional.

\section{LA LEGALIDAD DE LAS INTERVENCIONES HUMANITARIAS}

La doctrina internacional ha desarrollado una serie de requisitos que nos van a permitir identificar una determinada intervención como humanitaria o no. ${ }^{3}$ Entre los referidos requisitos, se deben destacar los siguientes: La existencia de una grave violación de los derechos fundamentales; el encontrarse en una situación de urgencia que haga imperiosa la necesidad de actuar; el agotamiento previo de los demás medios de protección sin que se haya conseguido salvaguardar esos derechos fundamentales; el respeto de la proporcionalidad entre el uso de la fuerza y los objetivos perseguidos; la existencia de un límite de tiempo y espacio para la realización de la operación humanitaria; el que se cuente con la autorización del Consejo de Seguridad o se informe inmediatamente de la intervención a dicho órgano $\mathrm{y}$, si es el caso, al organismo regional competente. ${ }^{4}$

3 Vid. Yves Sandoz, "Límites y condiciones del derecho de intervención humanitaria. Derecho de intervención y derecho internacional en el ámbito humanitario. Hacia una nueva concepción de la soberanía nacional", Bruselas, 1994. (Ponencia)

4 Vid. Consuelo Ramón Chornet, Violencia Necesaria. La Intervención Humanitaria en Derecho Internacional, Ed. Trotta. Madrid, 1995. p. 61. 
Al respecto, se debe precisar que el Estado que lidera una fuerza multinacional de intervención o la organización regional que intervenga debe perseguir la detención inmediata de las violaciones de los derechos fundamentales básicos que se han producido hasta antes de la intervención o que puedan continuar durante su realización. Igualmente, los medios empleados para la intervención siempre deben inspirarse en los derechos fundamentales, situación que no siempre se da. Así, por ejemplo, cuando la Organización del Tratado del Atlántico Norte anunció que bombardearía la zona de conflicto en la ex -Yugoslavia, el simple anuncio generó una grave crisis humanitaria.

En el siguiente apartado se analizará, de manera detallada, el rol que cumple la protección de los derechos fundamentales cuando se decide llevar a cabo una determinada intervención humanitaria en el ámbito de la Sociedad Internacional.

\section{LA PROTECCIÓN DE LOS DERECHOS FUNDAMENTALES COMO FINALIDAD DE LA INTERVENCIÓN HUMANITARIA}

Sobre el particular, se quiere señalar que solamente las intervenciones humanitarias que se fundamenten en una real y efectiva protección de los derechos fundamentales tendrán asegurada su aplicación, ya que dichos derechos no reconocen limitación alguna en el tema de la soberanía y de las fronteras estatales. Así mismo, se puede señalar que

“[...] así como no es coactiva una simple declaración de un ministro criticando la ausencia de instituciones democráticas en otro Estado, tampoco pertenece a la jurisdicción interna de los Estados: el genocidio, el racismo, la deportación en masa de colectivos humanos."

Existe un argumento a favor de la doctrina de intervención humanitaria, en virtud del cual, si bien los Estados pueden reglarse a sí mismos y no ser interferidos por otros, ellos no tienen una plena autonomía, por cuanto no pueden aplicar cualquier tipo de política en el ámbito internacional, no pueden extender su territorialidad a otras unidades similares, ni vulnerar los derechos fundamentales de sus nacionales ${ }^{6}$.

Al respecto, se debe considerar que la mejor intervención es aquella que aparente

5 Alfonso Ruiz Miguel, "Soberanía e Intervención Bélica Humanitaria”. En: Roberto Bergalli y Eligio Resta, (Compiladores), Soberanía: Un Principio que se Derrumba. Aspectos metodológicos y jurídico-políticos. Paidós Ibérica, Barcelona, 1996, p. 59.

6 En opinión de Michael Ignatieff, el Estado-Nación sigue siendo el marco principal para la protección de los derechos humanos, por cuanto los regímenes constitucionales son su mejor garantía, ya que impiden la tiranía y la anarquía. Vid. Michael Ignatieff, Human Rights as Politics and Idolatry, Princeton, Princeton University Press, 2001. 
no serlo y en donde al no existir un consenso interno entre la población y sus gobernantes, el llevar a cabo la intervención humanitaria resulta indispensable.? Asimismo, se puede promover una intervención humanitaria, sobre la base de la defensa de los derechos sociales básicos (seguridad y subsistencia), en la medida que la custodia de los derechos antes referidos, es exigible al Estado. ${ }^{8}$ También, las intervenciones humanitarias pueden llevarse a cabo cuando los países se encuentran gobernados por representantes internacionalmente ilegítimos, en cuyo caso, se considera que es necesaria la aprobación de la población para actuar a través de una intervención, ya que, en caso contrario se vulneraría la soberanía estatal ${ }^{9}$. Siguida una línea de planteamiento similar se puede señalar que las intervenciones humanitarias, se llevan a cabo ante la necesidad de defender a los ciudadanos cuando ellos son víctimas inocentes y sus derechos fundamentales (vida, integridad física, libertad de conciencia, asociación y propiedades, entre ottros) son vulnerados. ${ }^{10}$

Tras esta exposición, queda en claro que la intervención humanitaria, supone la actuación corporativa de toda la Sociedad Internacional, condicionada siempre a la evaluación del contexto en el que se producirá y que no debe terminar por convertir un pretexto moral en excusa, donde unas veces se invoque y otras no, donde unas veces se aplique y otras no. Tanto es así, que como reconocen todos los promotores de esta idea, la intervención humanitaria está tan ligada al equilibrio de poder, que ella sólo ha podido hacerse realidad tras el fin de la Guerra Fría.

Según parte de la Doctrina, la intervención es una expresión de la ausencia de universalidad de los derechos fundamentales y una constatación de que se habla de una fuerza que depende de un equilibrio y del poder de los Estados. Desde nuestro punto de vista, la vigencia universal de los derechos fundamentales en las relaciones internacionales aún es un ideal inconcluso, pese a los avances logrados hasta la fecha. Lo peculiar de este motivo es que esa ausencia de normatividad universal, obliga a aceptar todas las excepciones, que tienen connotaciones, ventajas y perfiles para las grandes y medianas potencias que desean intervenir. Igualmente, un segundo aspecto controvertido, se relaciona con el factor discrecional particular, ese análisis, caso a caso, que no siempre es muy objetivo. Las grandes potencias suelen envolver sus intereses particulares en el lenguaje de los principios universales, para persuadir a los demás de que la intervención es conforme al ordenamiento jurídico internacional.

Una vez establecidos los aspectos vinculados al concepto, la legalidad y la protección de los derechos fundamentales en el ámbito de las intervenciones humanitarias, resulta conveniente analizar la controversia que surge entre la

7 Vid. Michael Walter, Guerras Justas e Injustas. Una Discusión Moral con Ilustraciones Históricas, Goyanarte, Editor. Buenos Aires, Argentina. 1980, p. 137.

8 Vid. David Luban, “Just War and Human Rights”, Philosophy and Public Affairs. Vol.9, No.2, 1980, pp.160-181.

9 Vid. Fernando Tesón, Humanitarian Intervention: An Inquiry into Law and Morality, Dobbs Ferry, NY, Transnational Publishers, 2d. Ed., 1988.

10 Vid. John Rawls," The Law of Peoples”, Critical Inquiry, Vol.20, No.1, 1993. 
implementación de intervenciones humanitarias y el principio de no intervención de los asuntos internos de otros países. A continuación se analiza dicho aspecto en detalle.

\title{
IV. EL PRINCIPIO DE NO INTERVENCIÓN EN LOS ASUNTOS INTERNOS DE OTROS PAÍSES VERSUS LA INTERVENCIÓN HUMANITARIA
}

En contraposición a la Resolución 2131 de la Asamblea General de las Naciones Unidas, de 1965 que establecía el Principio de no intervención en los asuntos internos de otros países, a fines del siglo pasado, en el seno de la Organización de las Naciones Unidas, se empezó a discutir de manera general sobre el derecho a intervenir en asuntos internos de otros países. ${ }^{11}$ En virtud de ello, durante el mandato de Boutros Boutros-Ghali, como Secretario General de las Naciones Unidas, se propició una drástica limitación de la soberanía que fue desarrollada en el documento denominado Una Agenda para la Paz. Así, por ejemplo, en la Recomendación No. 27 de dicho Informe, se señaló que

\begin{abstract}
"la época de la soberanía nacional, entendida como una atribución absoluta ya había pasado y que se necesitaba equilibrar las necesidades domésticas con un mundo crecientemente interdependiente". ${ }^{12}$
\end{abstract}

Posteriormente, este tema nuevamente fue desarrollado durante la LIV Sesión de la Asamblea General de las Naciones Unidas, llevada a cabo el 20 de septiembre de 1999, en donde se produjo un inusual debate sobre la soberanía de los Estados y el derecho a las intervenciones colectivas. Fue en esta oportunidad que el entonces Secretario General de la Organización de las Naciones Unidas, Kofi Annan, afirmó la Doctrina de la intervención humanitaria si los derechos fundamentales eran violados de modo evidente en un Estado determinado. En efecto, al reflexionar sobre la intervención en Timor Oriental, donde la Organización de las Naciones Unidas era garante del plebiscito para su independencia, Annan hizo un dolido contraste con la masacre de 500.000 ruandeses en 1994 cuando la inacción del Consejo de Seguridad impidió tomar medidas al respecto. En contraposición a ello, afirmó que una era global exige intervenciones en las cuales debía coincidir el interés colectivo con los intereses nacionales y de esta manera las violaciones masivas y sistemáticas de los derechos fundamentales no serían más toleradas.

11 En el marco de las Naciones Unidas, con la finalidad de establecer una compatibilidad entre la protección de la población civil y la soberanía nacional, se aprobaron por la Asamblea General de las Naciones Unidas, la Resolución A/43/131 de 8 de diciembre de 1988 y la Resolución A/45/100 de 14 de diciembre de 1990.

12 Vid. UN, An Agenda for Peace. Preventive Diplomacy Peacemaking and Peacekeeping. Report of the Secretary-General, 31-I-1992. A/47/277-S/2411. 
Así mismo, indicó que para poner en marcha esta nueva doctrina era necesario implementar el principio de la legitimidad universal y de la eficacia de la defensa de los derechos fundamentales, a través de los cuales, la Sociedad Internacional quedaría vinculada. Igualmente, para completar su doctrina, Annan añadió a lo antes referido, que si los Estados proclives a cometer acciones criminales tomaban conocimiento de que sus fronteras no representaban una defensa absoluta, y que el Consejo de Seguridad podía adoptar respecto de ellos medidas para detener los crímenes contra la humanidad, no se embarcarían tan fácilmente en ese curso de acciones con la esperanza de quedar impunes.

Al respecto, se debe señalar que el carácter prescriptivo de esta doctrina era coincidente con el discurso del Ministro de Relaciones Exteriores de Francia en aquel entonces, Lionel Jospin, quien afirmó que la Comunidad Internacional debía imponer la tesis de la soberanía limitada. A su vez, señaló que

"La misión de la Organización de las Naciones Unidas no se limitaba solamente a la solución de conflictos entre los Estados, ya que se extendía hacia la defensa de la dignidad humana en el seno de cada uno de ellos y, de ser necesario, contra los mismos Estados".

En esa Sesión, el ex-Presidente Clinton criticó la Doctrina de la Intervención Humanitaria, alegaba que apoyaba las injerencias unilaterales cuando se ponía en riesgo la credibilidad de la Sociedad Internacional para actuar en ciertos casos de violaciones graves a los derechos fundamentales; sin embargo, a continuación señaló, que los Estados Unidos de Norteamérica no podía hacer todo en todos los sitios; pero, que eso no significa que fueran indiferentes ante la destrucción de inocentes en cualquier parte del mundo; con lo cual, dejaba abierta para su país, la posibilidad de llevar a cabo posibles intervenciones humanitarias. En la actualidad se ha podido comprobar cómo los Estados Unidos de Norteamérica han utilizado dicha posibilidad durante sus actuaciones en Libia.

Por su parte, el vocero ruso en aquella Sesión de la Organización de las Naciones Unidas, Igor Ivanov (entonces Canciller), refiriéndose a la rebelión en Chechenia y al fundamentalismo islámico en la Federación Rusa advirtió que Naciones Unidas debía

"defender con fuerza los principios de la soberanía, la integridad territorial y la inviolabilidad de las fronteras nacionales."

Al respecto, se debe señalar que en la actualidad, Moscú aún promueve y alega el principio de no intervención en los asuntos internos, con la finalidad de que ningún Estado u organización internacional (Naciones Unidas, Organización del Tratado del Atlántico Norte, Unión Europea, entre otras), intervenga en los conflictos que se producen en los países que anteriormente integraban la ex-Unión de Repúblicas 
Socialistas Soviéticas (URSS) y a las que Moscú considera dentro del ámbito de su frontera próxima o eje de influencia. ${ }^{13}$

Se ha querido presentar los planteamientos de tres de los más importantes actores de la Sociedad Internacional durante la Guerra Fría, con la finalidad de demostrar que en el escenario internacional, los Estados actúan en función de sus intereses y sin importar el sufrimiento de los pueblos que se ven afectados gravemente en la vigencia de sus derechos fundamentales, por el simple hecho de encontrarse o de haber nacido en una zona convulsionada por conflictos que en la actualidad han dejado de ser entre Estados y son, cada vez, más de naturaleza interna y principalmente motivados por aspectos religiosos, raciales, ideológicos u otros.

En el siguiente apartado se analizará el rol de los Estados miembros de la Sociedad Internacional en lo relativo a la implementación de una intervención humanitaria, ya que la vulneración de los derechos fundamentales y del derecho internacional humanitario es un tema que reclama una reacción inmediata y contundente de la Sociedad Internacional.

\section{LA RESPONSABILIDAD COLECTIVA DE LOS ESTADOS Y LA IMPLEMENTACIÓN DE LAS INTERVENCIONES HUMANITARIAS}

Las intervenciones humanitarias al ser una manifestación de la responsabilidad colectiva de los Estados en la Sociedad Internacional, encuentran un importante soporte jurídico en el Artículo 1 de los Convenios de Ginebra de 1949, en la medida que en dicha disposición normativa, se establece el compromiso de respetar y de hacer respetar las disposiciones del Convenio de Ginebra en todas las circunstancias. Asimismo, dicha materia también ha sido desarrollada en el Artículo 1 apartado 4 del Protocolo Adicional I a los Convenios de Ginebra de 1977, en donde se establece la obligación de respetar y hacer respetar las disposiciones humanitarias y los derechos fundamentales durante los conflictos internacionales y de carácter no internacional, en la medida en que estos últimos estén cubiertos por el Artículo 3 Común a los Cuatro Convenios ${ }^{14}$.

13 Osetia del Sur, Abjasia, Chechenia y la zona del Transniester en Moldavia, son prueba de que la Federación Rusa aún persiste en la primacía de la soberanía incluso sobre el respeto de los derechos humanos. Vid. Antonio Manrique de Luna, "Der Konflikt in der Seperatistenregion von Südossetien (Georgien)“, BOFAXE, No.325D, Institut für Friedenssicherungsrecht und Humanitäres Völkerrecht, 15.08.2008; Michael Croft, "Russia’s Peacekeeping Policy: Differences in Approaches and Obstacles", Peacekeeping \& International Relations, September/October 1996, Vol.25, Issue 5, pp.5-7; A. Racvsky and I.N.Vorob'ev, Russian Approaches to Peacekeeping Operations, New York, United Nations Publications, 1994, p.6.

14 Aunque los conflictos sin carácter internacional, tal como están definidos por el Protocolo Adicional II no están explícitamente cobijados por la obligación de respetar y hacer respetar, sin embargo, puede considerarse que caen dentro del ámbito de esta disposición, por cuanto el Protocolo II es simplemente una elaboración del Artículo 3 común a los cuatro Convenios de Ginebra. 
Al respecto, se debe indicar que la obligación que han asumido los miembros de la Sociedad Internacional durante los conflictos internacionales e internos, tiene una doble dimensión, pues les exige el respetar y el hacer respetar los Convenios de Ginebra. En tal sentido, el respetar, significa que el Estado tiene la obligación de hacer todo lo que pueda para garantizar que sus órganos y todos los que estén bajo su jurisdicción respeten las normas en cuestión. Por su parte, el hacer respetar, significa que los Estados, estén o no en situación de conflicto, tienen que tomar todas las medidas posibles para garantizar que todos, en particular las partes en conflicto, respeten las normas.

En tal sentido, la Corte Internacional de Justicia, ha reforzado esta aseveración en su Opinión Consultiva sobre la Licitud de la amenaza o del empleo de armas nucleares $^{15}$, en donde señala que muchas de las normas del Derecho internacional humanitario aplicable en los conflictos armados son tan fundamentales que deben ser observadas por todos los Estados hayan o no ratificado los Convenios que las contienen. Igualmente, la Corte Internacional de Justicia, demostró en su decisión sobre la base jurídica de la causa en el caso Nicaragua ${ }^{16}$, que la obligación a la que se aludía en el Artículo 1 común a los cuatro Convenios de Ginebra, formaba parte del Derecho internacional consuetudinario. Por su parte, el Tribunal Penal Internacional para la ex-Yugoslavia, en su sentencia del 14 de enero de 2000, señaló que

"(como) consecuencia de su carácter absoluto, estas normas de derecho internacional humanitario (...) establecen obligaciones hacia la Sociedad Internacional en su conjunto, lo que tiene como consecuencia que cada miembro de la Sociedad Internacional tenga un interés jurídico en su observancia y, por consiguiente, tenga el derecho legítimo a exigir el respeto de dichas obligaciones." 17

Por todo lo referido, se puede indicar que los Convenios de Ginebra y su Protocolo Adicional I, proporcionan los medios necesarios para que los Estados puedan cumplir su obligación no sólo de respetar, sino también de hacer respetar el Derecho internacional humanitario en todas las circunstancias, por ello, la importancia de este artículo dentro de lo que definimos como intervenciones humanitarias. Asimismo, en el orden internacional actual, el derecho de los Estados debería permanecer dentro del ámbito de la Carta de las Naciones Unidas, es decir, respetar las disposiciones de su Capítulo VII (Acción en caso de amenazas a la paz, quebrantamientos de la paz o actos de agresión) y su Capítulo VIII (Acuerdos Regionales). Igualmente, se debe tener presente que las vulneraciones graves de los derechos fundamentales y del derecho internacional humanitario en los conflictos armados, pueden representar

15 Legality of the Threat or Use of Nuclear Weapons, Advisory Opinion, 8 de Julio de 1996, I.C.J. Reports, 1996, paragraph 79.

16 Military and Paramilitary Activities in and against Nicaragua (Nicaragua v. United States of America), Merits. Fallo del 27 de junio de 1986. I.C.J. Reports, 1996, párrafo 220.

17 The Prosecutor v. Zoran Kupreskic and Others, TPIY, Sala de Primera Instancia, Sentencia, La Haya, 14 de enero de 2000, Caso No. IT-95-16-T, párrafo 519. 
una amenaza para la paz y la seguridad internacional y que, por lo tanto, podrían desencadenar una acción coercitiva de las Naciones Unidas, de organizaciones regionales o de los Estados que lideran fuerzas multinacionales, en la medida que actúen dentro del marco de la Carta de San Francisco. Además cabe indicar que quienes intervienen en nombre de la Sociedad Internacional, con el objeto de sostener el respeto de los derechos fundamentales y del derecho internacional humanitario, quedan sujetos en su actuación por este último. ${ }^{18}$

En el siguiente apartado se abordará de manera detallada la responsabilidad de proteger los derechos fundamentales que tienen todos los Estados miembros de la Sociedad Internacional y en virtud de lo cual participan en la realización de intervenciones humanitarias.

\section{LA RESPONSABILIDAD DE PROTEGER COMO FUNDAMENTO DE UNA INTERVENCIÓN HUMANITARIA}

Desde que se autorizara por el Secretario General y la Asamblea General de Naciones Unidas, al gobierno de Canadá para que creara una Comisión Internacional sobre Intervención y Soberanía de los Estados en el año 2000, diversas personalidades de todo el mundo y de distintas procedencias orgánicas, trabajaron durante dos años hasta que a finales de 2001, emitieron el Informe sobre la Responsabilidad de Proteger $^{19}$; sin embargo, en un escenario internacional convulsionado por los trágicos acontecimientos del 11-S, su aparición pasó desapercibida.

Respecto del Informe sobre la Responsabilidad de Proteger, se debe señalar que dicha responsabilidad presenta una triple dimensión: La responsabilidad de prevenir, la de reaccionar y la de reconstruir y, se encuentra sustentado en dos principios básicos, en virtud de los cuales el Estado debe proteger a su población y si él no lo hace, la Sociedad Internacional, tiene la obligación de intervenir para evitar genocidios y violaciones masivas de los derechos fundamentales. Igualmente, el Informe trata de responder aspectos fundamentales de la intervención humanitaria como son: cuándo intervenir, cómo hacerlo, quién debe autorizarlo, quién debe ejecutarlo y por cuánto tiempo, entre otros aspectos.

A continuación se analiza cada una de las dimensiones del tridimensional deber de proteger: La responsabilidad de prevenir, la de reaccionar y la de reconstruir.

\section{VI.I. La responsabilidad de prevenir}

Esta dimensión del deber de proteger, implica el eliminar tanto las causas profundas como las causas directas de los conflictos internos y de otras crisis provocadas por el hombre que pongan en peligro a la población. Al respecto, el

18 Boletín del Secretario General, Observancia del Derecho Internacional Humanitario por las Naciones Unidas, 6 de agosto de 1999, ST/SGB/1999/13

19 Loc. Cit. 
informe precisa algunos esfuerzos que deben hacerse para evitar las causas de ciertos conflictos (luchar contra la pobreza, las rivalidades étnicas y otras); sin embargo, el informe muestra una gran ambigüedad respecto de cómo resolver esos problemas. Así, por ejemplo, en su punto 3.22 sostiene que para resolver las causas profundas de un conflicto, se requiere resolver el tema de la pobreza y la falta de oportunidades económicas; pero olvida desarrollar temas básicos como el Estado de Bienestar ${ }^{20}$, la redistribución y el comercio justo. Desde nuestro punto de vista, si no se atienden estos ámbitos existirán conflictos en los países no desarrollados.

\section{VI.II. La responsabilidad de reaccionar}

En lo relativo a este aspecto, se establece que la intervención militar con fines de protección humana es una medida excepcional y extraordinaria. Añadir que se debe intervenir cuando hay o se estima que habrá pérdidas de vida a gran escala; y cuando, hay o se estima que habrá persecución étnica.

Al respecto, el Informe sobre la Responsabilidad de Proteger, precisa que la orden para intervenir en un conflicto por razones humanitarias, la debe dar el Consejo de Seguridad de las Naciones Unidas y que dicha orden debe ser previa a la intervención y evaluada a la luz de los hechos y justificaciones para que se lleve a cabo. Asimismo, el referido Informe, propone a los miembros permanentes que no usen su derecho a veto cuando sus intereses vitales no se viesen afectados y establece que en caso de que el Consejo de Seguridad no acepte una propuesta de intervención o no la examine en un plazo razonable, se tomen las siguientes medidas alternativas:

- Que la Asamblea General examine la cuestión en un período extraordinario de sesiones de emergencia, con arreglo al procedimiento establecido en la Resolución "Unión por la Paz"21; y

- Que una organización regional o subregional, en virtud de lo dispuesto en el Capítulo VIII de la Carta de la ONU, actúe dentro de su zona de jurisdicción y posteriormente solicite la autorización del Consejo de Seguridad.

A manera de crítica, se puede señalar que el Informe denominado "La responsabilidad de Proteger", no se pronuncia sobre la falta de representación de los países en vías de desarrollo en el seno del Consejo de Seguridad y, por ende, deja sin abordar un tema fundamental que está relacionado directamente con el hecho de otorgar una mayor legitimidad a las intervenciones de las Naciones Unidas, de las organizaciones regionales y de los Estados que lideran fuerzas multinacionales; ya que a través de una participación más relevante de los países en vías de desarrollo, se podría lograr un mayor consenso en el despliegue de una fuerza de intervención por razones humanitarias.

20 La experiencia ha demostrado en los casos de Europa, Asia y Estados Unidos que no existe desarrollo sustentable sin la intervención del Estado en la educación, salud y otros.

21 Vid. Resolución de la Asamblea General de las Naciones Unidas $A / R E S / 377(V)$, de 3 de noviembre de 1950. 


\section{VI.III. La responsabilidad de reconstruir}

Desde nuestro punto de vista, en este ámbito, el referido Informe hace un desarrollo adecuado de la reconstrucción de un Estado que ha estado afectado por un conflicto, al abordar temas como la reconciliación nacional, el desarrollo y la transición de las autoridades desde el ámbito internacional al local. Sin embargo, no se pronuncia respecto del rol de la población civil (principal víctima de los conflictos), durante este período de reconstrucción; aunque sí lo hace respecto de la relación entre los actores internacionales y la autoridad local. Si se toma en consideración esta ausencia de referencia al rol de la población civil durante la etapa de reconstrucción, se cree que en esta etapa, la población civil, puede desempeñar un rol muy importante en los siguientes ámbitos:

- Monitorear la transición de autoridad desde el ámbito internacional al local, al controlar que la ayuda humanitaria llegue a la población ${ }^{22}$;

- Denunciar ante la justicia las vulneraciones de los derechos fundamentales cometidos durante el período de crisis; y

- Controlar el accionar de las fuerzas de seguridad post-conflicto.

Por ello, se cree que es necesario un diálogo fluido entre la Organización de las Naciones Unidas y la población civil para fortalecer el proceso de reconstrucción, ya que de esa manera, la organización mundial, se mantendría informada de los diversos acontecimientos que se produzcan durante el despliegue de las fuerzas de intervención por razones humanitarias y, con ello, podría elaborar una mejor planificación respecto de la reconstrucción y el restablecimiento de la plena vigencia de los derechos fundamentales en aquella zonas devastadas por los conflictos.

Asimismo, se debe señalar que los planteamientos que se esbozaron en el Informe sobre la Responsabilidad de Proteger, han sido reafirmados por los trabajos del Grupo de alto nivel sobre las amenazas, los desafíos y el cambio ${ }^{23}$ (2004); por el Informe que emitió el Secretario General de las Naciones Unidas bajo el título "Un concepto más amplio de la libertad: desarrollo, seguridad y derechos humanos para todos"24 (2005) y; por el Documento Final de la Cumbre Mundial (2005), en donde los representantes de las Naciones Unidas, se pronunciaron respecto de esta problemática, bajo los siguientes términos:

22 En inglés recibe la denominación de Accountability.

23 Ese grupo de trabajo, dio una gran importancia a los aspectos vinculados con la autorización que debe dar el Consejo de Seguridad de las Naciones Unidas, para que una intervención humanitaria pueda emplear la fuerza durante su despliegue, en la medida que sea el último recurso disponible y que se respete el principio de proporcionalidad. Vid. Naciones Unidas, "Informe del Grupo de alto nivel sobre las amenazas, los desafíos y el cambio". Resolución A/59/565 de 2 de Diciembre de 2004.

24 Vid. Naciones Unidas, "Un concepto más amplio de la libertad: desarrollo, seguridad y derechos humanos para todos". Informe del Secretario General de las Naciones Unidas. Resolución A/59/2005 de 21 de marzo de 2005. 
"Cada Estado es responsable de proteger a su población del genocidio, los crímenes de guerra, la depuración étnica y los crímenes de lesa humanidad. Esa responsabilidad conlleva la prevención de dichos crímenes, incluida la incitación a su comisión, mediante la adopción de las medidas apropiadas y necesarias.

Aceptamos esa responsabilidad y convenimos en obrar en consecuencia. La comunidad internacional debe, según proceda, alentar y ayudar a los Estados a ejercer esa responsabilidad y ayudar a las Naciones Unidas a establecer una capacidad de alerta temprana.

La comunidad internacional, por medio de las Naciones Unidas, tiene también la responsabilidad de utilizar los medios diplomáticos, humanitarios y otros medios pacificos apropiados, de conformidad con los Capitulos VI y VIII de la Carta, para ayudar a proteger a las poblaciones del genocidio, los crímenes de guerra, la depuración étnica y los crímenes de lesa humanidad. En este contexto, estamos dispuestos a adoptar medidas colectivas, de manera oportuna y decisiva, por medio del Consejo de Seguridad, de conformidad con la Carta, incluido su Capítulo VII, en cada caso concreto y en colaboración con las organizaciones regionales pertinentes cuando proceda, si los medios pacificos resultan inadecuados y es evidente que las autoridades nacionales no protegen a su población del genocidio, los crímenes de guerra, la depuración étnica y los crímenes de lesa humanidad (...)” 25

En la actualidad, las Naciones Unidas en virtud de las disposiciones de la Carta de San Francisco, de las diversas obligaciones jurídicas que emanan de las declaraciones, pactos y tratados relativos al Derecho Internacional Humanitario, los derechos humanos y el derecho interno, aún trabaja en el ámbito de la responsabilidad de proteger, para establecer con claridad sus características, naturaleza jurídica y contenido. Se cree que ello es fundamental, ya que de esta manera, se busca evitar un uso arbitrario e ilegal de una importante herramienta que la ingeniería jurídica internacional, ha creado para contribuir a frenar las vulneraciones de los derechos fundamentales de las personas que se viven en zonas de conflicto.

A continuación se estudian las principales intervenciones humanitarias que se han llevado a cabo desde finales del siglo XX hasta la actualidad, con la finalidad de hacer un breve recuento de los aciertos y errores cometidos durante la realización de tales actividades.

\section{LA IMPLEMENTACIÓN PRÁCTICA DE LAS INTERVENCIONES HUMANITARIAS}

Durante el siglo pasado y el presente, se han producido diversas intervenciones con fines humanitarios. Algunas han resultado exitosas y otras han fracasado. A continuación se presentan algunas intervenciones que consideramos importantes.

25 Vid. Naciones Unidas, Documento Final de la Cumbre Mundial 2005. Resolución A/RES/60/1 de 24 de octubre de 2005, párrafos 138-139. 
En primer lugar, se debe hacer referencia a la intervención humanitaria que tuvo lugar en Irak con la finalidad de proteger al pueblo kurdo iraquí (1991), que fue víctima de las masacres ordenadas desde el régimen de $\mathrm{S}$. Hussein y que hicieron que el Consejo de Seguridad de las Naciones Unidas emitiera la Resolución 688, en virtud de la cual, se exigía a Irak que pusiera fin a esa represión y restableciera el respeto de los derechos humanos en su territorio. Desde nuestro punto de vista, esta Resolución marcó el inicio de la preocupación de la Sociedad Internacional, respecto de la situación que vivía un determinado pueblo que veía vulnerado sus derechos humanos.

En segundo lugar, se quiere analizar la intervención humanitaria que tuvo lugar en Haití (1991), con la finalidad de frenar la vulneración de los derechos fundamentales que se habían producido debido a los problemas democráticos que hicieron que su Primer Ministro se ausentara por más de 18 meses del ejercicio del poder. Como parte de la intervención humanitaria, se estableció una Misión Civil InternacionalMICIVIH y una Misión de Policía Civil de las Naciones Unidas en Haití- MIPONUH, con la finalidad de restablecer la libertad y respeto de los derechos humanos, así como también para restablecer la democracia. Sobre dicha intervención, se puede indicar que fue exitosa y que logró alcanzar los objetivos trazados.

En tercer lugar, se debe mencionar la intervención humanitaria en Somalia (1991), que buscaba frenar la anarquía casi total que se vivía en aquel país como consecuencia de las actividades del Movimiento Nacional Somalí que había proclamado la independencia del Noroeste del país, mientras que en el sur de su territorio estallaron conflictos étnicos. Ante tales circunstancias, la Organización de las Naciones Unidas en 1992 y en 1993 desplegó dos operaciones de paz ONUSOM I y II. Sin embargo, en 1995, dicha intervención terminó con un rotundo fracaso, principalmente debido a que existían líderes de facciones sin interés en la paz, clanes sin afán de reconciliación y milicias armadas que atizaban los conflictos.

En cuarto lugar, se refiere a la intervención humanitaria que tuvo lugar en la exYugoslavia, en donde debido a la catástrofe humanitaria que se produjo en Kosovo, se demostró cierta incapacidad del Consejo de Seguridad de las Naciones Unidas para gestionar las crisis internacionales. Ante dicha situación, el 23 de marzo de 1999, el entonces Secretario General de la OTAN, Javier Solana, tras realizar las consultas pertinentes, anunció el inicio de la operación "Fuerza Aliada", señaló que era un deber de la OTAN, el prevenir los sufrimientos, represión y violencia que afectaba a la población civil de Kosovo. Sin embargo, dicha actuación, no mitigó la catástrofe, pues, llegó en algunos momentos a incrementarla.

En quinto lugar, se debe indicar que la intervención humanitaria en Ruanda que fue autorizada mediante la Resolución 929 de 22 de junio de 1994, permitió la utilización de todos los medios necesarios para asegurar de forma imparcial la protección de las personas desplazadas, refugiados y civiles en Ruanda. La operación de carácter humanitaria que fue desplegada, no impidió las diversas matanzas que se produjeron en los campos de refugiados. Se llegó incluso a denunciar por diversas 
organizaciones humanitarias, la indiferencia de los cascos azules de la ONU ante tales crímenes.

Por su parte, la intervención de la OTAN en Kosovo en 1999 y la intervención británica en Sierra Leona en el 2000 se produjeron ante el riesgo inminente de una catástrofe humanitaria y con la aceptación de la Sociedad Internacional, lo cual convirtió a dichas acciones en justificadas y legítimas. En el caso de la intervención en Irak, la catástrofe humanitaria fue provocada por la guerra, ya que la invasión y la ocupación de Irak requerían de un mandato explícito del Consejo de Seguridad, cosa que el gobierno de Estados Unidos de Norteamérica, no estuvo en condiciones de obtener.

Finalmente, la intervención humanitaria en Libia, que fue aprobada por la Resolución 1973 de 17 de marzo de 2011, con la finalidad de proteger a los civiles y las zonas pobladas por civiles que estén bajo amenaza de ataque en Libia, incluida Benghazi, constituye un ejemplo de una intervención humanitaria que no ha logrado frenar las vulneraciones de los derechos fundamentales existentes y que pese a encontrarse en la actualidad bajo el mando de la Organización del Tratado del Atlántico Norte que finalmente sustituyó el liderazgo confuso e inicial de los Estados Unidos de Norteamérica, Francia y Reino Unido, lamentablemente no se puede indicar que las vulneraciones de los derechos fundamentales hayan sido frenadas gracias a la actuación de la intervención humanitaria.

\section{CONCLUSIÓN}

El llamado derecho de injerencia humanitaria o las denominadas intervenciones humanitarias, deben contar con la correspondiente autorización del Consejo de Seguridad de las Naciones Unidas, ya que en caso contrario, dichas operaciones serían llevadas a cabo fuera del marco del Derecho internacional vigente. Así mismo, la delegación por el Consejo de Seguridad de las Naciones Unidas de facultades o poderes de intervención que son otorgados a un Estado que lideran fuerzas multinacionales o a una organización regional para llevar a cabo acciones coactivas, de ninguna manera está exenta de la referida autorización, ya que el preservar la paz y seguridad internacional constituyen, por definición, cometidos esenciales e irrenunciables del sistema internacional con base en la Carta de Naciones Unidas y en el Derecho internacional público.

A su vez, el responder con medidas rápidas y eficaces a todas aquellas situaciones conflictivas en donde se produzca una violación flagrante de los derechos fundamentales, constituye una obligación de la Sociedad Internacional. No obstante ello, se debe tener presente que la intervención humanitaria está diseñada para contemplar las consecuencias, pero no las causas de los conflictos. En otras palabras, no está diseñada para resolver los conflictos, ya que su único objetivo es proteger la dignidad humana y salvar vidas. 
Respecto del marco jurídico de las intervenciones humanitarias, se ha comprobado el importante rol desempeñado por el Artículo 1 Común a los Cuatro Convenios de Ginebra de 1949, que ha permitido desarrollar un sistema de responsabilidad colectiva, basado en un enfoque tridimensional. Donde, el deber de proteger, implica la responsabilidad de prevenir, la de reaccionar, y la de reconstruir, para que de esta manera no se produzcan conflictos donde los derechos fundamentales son constantemente vulnerados.

\section{REFERENCIAS}

\section{TESIS}

Danish Institute Of International Affairs, (1999) Humanitarian Intervention, Legal and Political Aspects, DUPI.

Ignatieff, Michael, (2001) Human Rights as Politics and Idolatry, Princeton, Princeton University Press.

Kofi, Francis, (1999) The Evolution of the Doctrine and Practice of Humanitarian Intervention, Kluwer Law International.

Racvsky, A. and VOROB'EV, I.N., (1994) Russian Approaches to Peacekeeping Operations, New York, United Nations Publications.

Ramón, Consuelo. (1995) Violencia Necesaria. La Intervención Humanitaria en Derecho Internacional, Ed. Trotta. Madrid.

Ruiz, Alfonso. (1996) "Soberanía e Intervención Bélica Humanitaria”. En: Roberto Bergalli y Eligio Resta, (Compiladores), Soberanía: Un Principio que se Derrumba. Aspectos metodológicos y jurídico-políticos. Paidós Ibérica, Barcelona.

Tesón, Fernando. (1988) Humanitarian Intervention: An Inquiry into Law and Morality, Dobbs Ferry, NY, Transnational Publishers, 2d. Ed.

Walter, Michael, (1980) Guerras Justas e Injustas. Una Discusión Moral con Ilustraciones Históricas, Goyanarte, Editor. Buenos Aires, Argentina.

\section{ARTÍCULOS EN PUBLICACIONES PERIÓDICAS}

Croft, Michael. (1996) "Russia's Peacekeeping Policy: Differences in Approaches and Obstacles", Peacekeeping \& International Relations, September/October 1996 Vol.25, Issue 5, pp.5-7.

Luban, David. (1980) “Just War and Human Rights”, Philosophy and Public Affairs, Vol.9, No.2. 
Manrique De Luna, Antonio., (2008) "Der Konflikt in der Seperatistenregion von Südossetien (Georgien)“, BOFAXE, No.325D, 15.08.

Rawls, John. (1993) " The Law of Peoples", Critical Inquiry, Vol.20, No.1.

Sorel, Jean-Marc. (1992) "La Somalia et les Nations Unies”, Annuaire Français de Droit Internacional, Vol. XXXVIII, pp.61-88.

\section{JURISPRUDENCIA INTERNACIONAL}

\section{Corte Internacional de Justicia}

Legality of the Threat or Use of Nuclear Weapons, Advisory Opinion, 8 de Julio de 1996, I.C.J. Reports, 1996, paragraph 79.

Military and Paramilitary Activities in and against Nicaragua (Nicaragua v. United States of America), Merits. Fallo del 27 de junio de 1986. I.C.J. Reports, 1996, párrafo 220 .

\section{Tribunal Penal Internacional para la antigua Yugoslavia}

The Prosecutor v. Zoran Kupreskic and Others, TPIY, Sala de Primera Instancia, Sentencia, La Haya, 14 de enero de 2000, Caso No. IT-95-16-T, párrafo 519.

\section{DOCUMENTOS Y DISPOSICIONES NORMATIVAS DE LA ONU}

Boletín Del Secretario General, Observancia del Derecho Internacional Humanitario por las Naciones Unidas. ST/SGB/1999/13 de 6 de agosto de 1999.

United Nations, An Agenda for Peace. Preventive Diplomacy Peacemaking and Peacekeeping. Report of the Secretary-General. A/47/277-S/2411, 31st of January 1992.

Naciones Unidas. Documento Final de la Cumbre Mundial 2005".A/RES/60/1 de 24 de octubre de 2005, párrafos 138-139

Naciones Unidas. Informe del Grupo de alto nivel sobre las amenazas, los desafíos y el cambio. A/59/565 de 2 de diciembre de 2004.

Naciones Unidas. Situación de la democracia y de los derechos humanos en Haití. A/52/687 de 18 de noviembre de 1997.

Naciones Unidas. Un concepto más amplio de la libertad: desarrollo, seguridad y derechos humanos para todos. A/59/2005 de 21 de marzo de 2005.

ONU. (1950). Resolución A/RES/377 ( $V$ ) de 3 de noviembre de 1950.

ONU. (1988). Resolución A/43/131 de 8 de diciembre de 1988.

ONU. (1990). Resolución A/45/100 de 14 de diciembre de 1990.

ONU. (2011). Resolución S/RES/ 1973 de 17 de marzo de 2011. 